\title{
Differentially expressed Maf family transcription factors, c-Maf and MafA, activate glucagon and insulin gene expression in pancreatic islet $\alpha$ - and $\beta$-cells
}

\author{
K Kataoka, S Shioda, K Ando', K Sakagami, H Handa ${ }^{1}$ and K Yasuda \\ Laboratory of Molecular and Developmental Biology, Graduate School of Biological Science, Nara Institute of Science and Technology, 8916-5 \\ Takayama-cho, Ikoma 630-0192, Japan \\ ${ }^{1}$ Frontier Collaborative Research Center, Tokyo Institute of Technology, Japan
}

(Requests for offprints should be addressed to K Kataoka; Email: kkataoka @bs.aist-nara.ac.jp)

\begin{abstract}
A basic-leucine zipper transcription factor, MafA, was recently identified as one of the most important transactivators of insulin gene expression. This protein controls the glucose-regulated and pancreatic $\beta$-cell-specific expression of the insulin gene through a cis-regulatory element called RIPE3b/MARE (Maf-recognition element). Here, we show that MafA expression is restricted to $\beta$-cells of pancreatic islets in vivo and in insulinoma cell lines. We also demonstrate that c-Maf, another member of the Maf family of transcription factors, is expressed in islet $\alpha$-cells and in a glucagonoma cell line ( $\alpha \mathrm{TC1}$ ), but not in $\gamma$ - and $\delta$-cells. An insulinoma cell line, $\beta$ TC6, also expressed c-Maf, albeit at a low level. Chromatin immunoprecipitation assays demonstrated that Maf proteins associate with insulin and glucagon promoters in $\beta$ - and $\alpha$-cell lines, respectively. c-Maf protein stimulated glucagon promoter activity in a transient luciferase assay, and activation of the glucagon promoter by c-Maf was more efficient than by the other $\alpha$-cell-enriched transcription factors, Cdx2, Pax6, and Isl-1. Furthermore, inhibition of c-Maf expression in $\alpha \mathrm{TC} 1$ cells by specific short hairpin RNA resulted in marked reduction of the glucagon promoter activity. Thus, $c$-Maf and MafA are differentially expressed in $\alpha$ - and $\beta$-cells where they regulate glucagon and insulin gene expression, respectively.
\end{abstract}

Journal of Molecular Endocrinology (2004) 32, 9-20

\section{Introduction}

Endocrine cells of the pancreatic islets of Langerhans are divided into four types $(\alpha, \beta, \delta$ and $\gamma$ ) depending on the hormones that they specifically secrete (glucagon, insulin, somatostatin or pancreatic polypeptide). These hormones play critical roles in the homeostasis of blood glucose levels, and the mechanisms of their specific expression have been extensively investigated. In particular, exclusive expression of insulin and glucagon in $\beta$ - and $\alpha$-cells, respectively, is known to involve a combination of multiple cell-type-specific transcription factors acting on their promoter regions (Drucker et al. 1987, Karlsson et al. 1987, Philippe et al. 1988, Crowe \& Tsai 1989, Whelan et al. 1989, Shieh \& Tsai 1991).

$\beta$-Cell-specific expression of insulin is mainly regulated through three conserved enhancer elements in the insulin promoter region termed $\mathrm{E} 1$, A3 and RIPE3b/C1 (Ohneda et al. 2000, Melloul et al. 2002). Two transcription factors, Beta2/ NeuroD and Pdx1/IPF1/STF1/GSF1/IUF1, whose expression is restricted to pancreatic islets and which bind to the E1 and A3 elements, respectively, have been isolated (Ohlsson et al. 1993, Petersen et al. 1994, Naya et al. 1995, Marshak et al. 1996). The RIPE3b element is quite similar to the binding sequence of Maf family transcription factors termed MARE (Maf-recognition element) (Kataoka et al. 1994b), and recently we and others have identified the RIPE3b-binding factor (RIPE3b1 activator) as MafA, a member of the Maf family (Kataoka et al. 2002, Olbrot et al. 2002, Matsuoka et al. 2003).

In contrast, $\alpha$-cell-specific expression of the glucagon gene is established by islet-specific enhancer elements (G2, G3 and G4) and an 
$\alpha$-cell-specific control region (G1) within the glucagon gene promoter region (Knepel et al. 1990, 1991, Morel et al. 1995). Islet-enriched transcription factors, Pax6, Gdx2 and Isl-1, have been shown to bind to the G1 region (Wang \& Drucker 1995, Jin \& Drucker 1996, Laser et al. 1996, Ritz-Laser et al. 1999). Pax6 also binds to the G3 element (Ritz-Laser et al. 1999). Recently, Large Maf family members were shown to bind to the MARE-like sequence in the G1 region (Planque et al. 2001).

Large Maf is a subgroup of the Maf family of basic-leucine zipper (bZip) transcription factors and includes MafA, MafB, c-Maf and Nrl. They possess transactivator domains in their amino-terminal regions and bZip domains in their carboxyterminal regions (Kawai et al. 1992, Swaroop et al. 1992, Kataoka et al. 1994a, 2002, Ogino \& Yasuda 1998, Olbrot et al. 2002). These two domains are well conserved among Large Maf members.

As mentioned above, accumulating evidence suggests that Large Maf members potentially play important roles in the regulation of cell-typespecific expression of insulin and glucagon genes. However, direct evidence of their involvement in this process is still lacking. Furthermore, it is still unknown which of the Large Maf members is expressed in each endocrine cell type of pancreatic islets. Here, we clarified that MafA and c-Maf are differentially expressed in $\beta$ - and $\alpha$-cells by defining the specificities of available anti-Maf antibodies. We also demonstrated that MafA and c-Maf directly bind to and regulate insulin and glucagon promoters in a cell-type-specific manner.

\section{Materials and methods}

\section{Cells}

$\alpha \mathrm{TC} 1$ clone 9 (hereafter $\alpha \mathrm{TC1}$ ) and $\beta$ TC6 were purchased from the American Tissue Culture Collection (Manassas, VA, USA). MIN6 cells (Miyazaki et al. 1990) were a generous gift of Dr Jun-ichi Miyazaki (Osaka University, Japan). $\alpha$ TC1 cells were grown in F12K medium supplemented with $10 \%$ fetal bovine serum, and $\beta$ TC6 and MIN6 cells were grown in Dulbecco's modified Eagle's medium supplemented with $15 \%$ fetal bovine serum. HeLa and NIH3T3 cells were grown in Dulbecco's modified Eagle's medium supplemented with $10 \%$ fetal bovine serum.

\section{Immunostaining}

Five-week-old mouse pancreas tissue was fixed overnight in 4\% paraformaldehyde, embedded in Optical Cutting Temperature compound (Sakura, Tokyo, Japan) and snap frozen. Cryostat sections were cut at $10 \mu \mathrm{m}$. Anti-c-Maf (M-153, rabbit), anti-c-Maf (N-15, goat), anti-insulin A (C-12, goat), anti-glucagon (N-17, goat), anti-somatostatin (D-20, goat) (Santa Cruz Biotechnology, Inc., Santa Cruz, CA, USA), and anti-pancreatic polypeptide (guinea pig) (Linco Research Inc., St Louis, MO, USA) were obtained from commercial sources. Anti-MafA sera have been described previously (Kataoka et al. 2002). Anti-MafB serum was obtained by immunizing rabbits with a keyhole limpet hemocyanin-conjugated synthetic peptide (KKEPLGRAERPGRPG) corresponding to the amino-terminal part of mouse MafB. Alexa Fluor 488-labeled anti-rabbit, goat or guinea pig IgG sera and Alexa Fluor 594-labeled anti-rabbit IgG serum (Molecular Probes, Inc., Eugene, OR, USA) were used as secondary antibodies. Immunostaining of cultured cells was performed as described previously (Kataoka et al. 2002). Mice were handled and used in accordance with the National Institute of Health Guide for the Care and Use of Laboratory Animals.

\section{Western blotting}

HeLa cells were transfected with expression vectors for enhanced green fluorescent protein (EGFP) (pHygEF2/EGFP), mouse MafA (pHygEF2/mmafA), mouse MafB (pHygEF2/m-mafB) or HAtagged human c-Maf, which has a serine substituted by asparagine at the eighth amino acid position (pHygEF2/HA-h-c-maf-SoN), using Lipofectamine 2000 reagent (Invitrogen). Human c-Maf-S8N was used as a substitute for mouse c-Maf because the eighth amino acid residue of human (serine) and of mouse (asparagine) c-Maf is the only species difference within the amino-terminal half (first 177 amino acids) of these two proteins.

Aliquots of total cell extracts of $\alpha \mathrm{TC} 1, \beta \mathrm{TC} 6$ or transfected HeLa cells were separated by $8 \%$ SDS-PAGE and transferred onto an Immobilon membrane (Millipore Corp., Bedford, MA, USA). The membrane was stained with anti-c-Maf (M-153), anti-c-Maf (N-15) (Santa Cruz Biotechnology), anti-MafA or anti-MafB sera, and then 
with horseradish peroxidase-conjugated anti-rabbit (Dako Corp., Carpinteria, CA, USA) or anti-goat IgG (Santa Cruz Biotechnology) secondary antibodies.

\section{Chromatin immunoprecipitation assay}

The chromatin immunoprecipitation assay was performed as described previously (Boyd et al. 1998). Anti-glutathione S-transferase (GST) (Santa Cruz Biotechnology) was used as a negative control for anti-c-Maf (M-153), and anti-MafA preimmune serum for anti-MafA and anti-v-Maf (Kataoka et al. 1993) sera. PGR primers used were as follows: insulin II promoter, 5'-GGCCCCTTG TTAAGACTCTAATTACGCTAG-3' and 5'-CGT GCTTGCTGATGGTTTTTGATTGTAGCG-3'; glucagon promoter, 5'-TAAAATGTTTCGGGGG CTCTGGGGTCTC- ${ }^{\prime}$ and 5'-AGTCGCGTT GGGAACTTTGAGTGTGTCG-3'; proliferating cell nuclear antigen (PCNA) promoter, 5'-CTAAA GTCGCACAAAGTTGGGGGGTGAC-3' and 5'AGGCGTACAGCGACAAGTACGAACGCTC-3'.

\section{Luciferase assay}

The mouse glucagon promoter region was amplified by PCR using primers $(5$ '-caggtaccTTTGAC AGTTTTTCGTGCCTG-3' and 5'-agaagCTTG GGAACTTTGAGTGTGTCGT-3') from Balb/c mouse genomic DNA. The fragment was digested with KpnI and HindIII, and was inserted into the KpnI-HindIII site of the pGL2-basic plasmid (Promega) to generate m-ggn-p-luc.

The expression plasmid for mouse mafA (pHygEF2/m-mafA) has been described previously (Kataoka et al. 2002). The mouse mafB open reading frame was excised from the mouse genomic clone of the mafB locus (pMmb32) (a generous gift from Dr Makoto Nishizawa, (unpublished observations)) by digestion with EarI, and inserted into the EcoRV site of pBluescript II SK( - ) in the T7-T3 direction. The pBluescript II $\mathrm{SK}(-) / m-m a f B$ plasmid was then digested with ClaI and SpeI, and the fragment was inserted into the $C l a \mathbf{I}-X b a \mathbf{I}$ site of the $\mathrm{pHygEF} 2$ expression vector to generate pHygEF2/m-mafB. A DNA fragment (Pst $\mathrm{I}-$ NheI) containing the human c-maf open reading frame was excised from human a genomic clone that encompasses the c-maf locus (pHM-3) (a generous gift from Dr Makoto Nishizawa, (unpublished observations)), and was inserted into the PstI-NheI site of pGEM3 (Promega) to generate pGEM3/h-cmaf. The plasmid was digested with $X b a \mathrm{I}$ and $\mathcal{N h e} \mathrm{I}$, and the resultant $h$-c-maf-containing fragment was inserted into $\mathrm{XbaI}$-digested $\mathrm{pHygEF} 2$ to generate pHygEF2/h-c-maf. The mouse $c d x 2$ open reading frame was amplified by RT-PGR from $\beta$ TC6 total RNA using primers 5'-CTCTGGGTCGCTCG CCACGATGTACGTG-3' and 5'-CACGGGAGG GGTCACTGGGTGACAGTGG-3', and inserted into pGEM-T-easy (Promega) by T/A cloning. The plasmid was digested with $\mathcal{N o t} \mathrm{I}$ and the fragment was inserted into the $\mathcal{N o t I}$ site of $\mathrm{pHygEF} 2$ to generate $\mathrm{pHygEF} 2 / m-c d x 2$. The Pax6 expression vector pCAGGS/Pax6 and pCAGGS/Pax6-5a were generous gifts from Dr Noriyuki Azuma (National Children's Hospital, Tokyo, Japan) and Dr Yuki Yamaguchi (Tokyo Institute of Technology, Yokohama, Japan) (Yamaguchi et al. 1997). Expression plasmids for FLAG-tagged mouse Isll splicing isoforms (pHygEF2/FLAGisl1- $\alpha$ and pHygEF2/FLAG-isl1- $\beta$ ) have been described previously (Ando et al. 2003).

An expression vector to express short hairpin RNA (shRNA) was designed to clone a doublestranded synthetic oligonucleotide into U6 small nuclear RNA promoter (Sui et al. 2002) using $B s e \mathrm{RI}-$ HindIII restriction sites. The oligonucleotides used were as follows: c-maf, 5'-ACTACTACT GGATGACGGttcaagagaCGGTCATCGAGTAG TAGTCttttt-3' and 5'-gatcaaaaaGACTACTACT GGATGACGGtctcttgaaCGGTCATCGAGTAG TAGTca-3'; mafB, 5'-TTGGAGGTGAAGAAGGA GttcaagagaCTCGTTCTTCACGTCGAACtttt-3' and 5'-gatcaaaaaGACGCATCTCGAGGACGTG tctc t tgaaCAGGTCGTCGAGATGGGTca-3'.

aTC1 or NIH3T3 cells grown in 12-well plates were transfected with a total of $1.6 \mu \mathrm{g}$ plasmid $(0 \cdot 1 \mu \mathrm{g}$ luciferase plasmid, $1 \cdot 4 \mu \mathrm{g}$ expression plasmid and $0 \cdot 1 \mu \mathrm{g}$ pEF-Rluc) using $4 \mu \mathrm{l}$ Lipofectamine 2000 (Invitrogen). Cells were harvested $24 \mathrm{~h}$ after transfection. Firefly and Renilla luciferase activities were measured using the Dual Luciferase Assay System (Promega).

\section{Results}

\section{Expression of Large Maf members in pancreatic islet endocrine cells}

We have previously shown that MafA protein is specifically expressed in mouse $\beta$-cell lines (insulinoma, 
MIN6 and $\beta$ TC6), but is not detected in an $\alpha$-cell line (glucagonoma, $\alpha \mathrm{TC} 1$ ) (Kataoka et al. 2002). However, it remained to be determined whether MafA expression is restricted to the $\beta$-cells of pancreatic islets in vivo. We therefore stained adult mouse pancreas with anti-MafA antiserum, which was raised against a MafA-specific peptide. As shown in Fig. 1A (top panels), most, but not all, nuclei of islets were clearly stained by the MafA serum. Double staining with antibodies to specific cell markers, anti-insulin ( $\beta$-cell) (Fig. 1A, left panels), anti-glucagon ( $\alpha$-cell) (Fig. $1 \mathrm{~A}$, second left panels), anti-somatostatin ( $\delta$-cell) (Fig. 1A, third left panels), or anti-pancreatic polypeptide $(\gamma$-cell $)$ (Fig. 1A, right panels), clearly revealed that MafA is selectively expressed in insulin-producing $\beta$-cells. MafA expression never overlapped with that of glucagon, somatostatin or pancreatic polypeptide.

We also used anti-c-Maf (M-153) antibody (Santa Gruz Biotechnology), which was raised against a c-Maf recombinant protein of 153 amino acids and was expected to cross-react with the other Large Maf family members, MafA, MafB and Nrl. As shown in Fig. 1B (top panels), nuclear staining of islet cells was observed with this antibody. Nuclei of some non-islet cells also stained positively (Fig. 1B, top panels, arrowheads), but the cell type of these cells remains to be determined. By double staining, we found that insulin-positive cells stained positively with anti-c-Maf (M-153). However, in contrast to anti-MafA staining, some cells positive for staining with anti-c-Maf (M-153) were negative for insulin (Fig. 1B, left panels, arrows) but were positive for glucagon (Fig. 1B, second left panels). These data indicated that c-Maf and/or immunologically related Large Maf proteins other than MafA are expressed in $\alpha$-cells. It is also possible that in addition to MafA, $\beta$-cells express other Large Maf proteins.

\section{Preferential expression of MafA and c-Maf in $\beta$ - and $\alpha$-cell lines}

In order to define which members of the Large Maf family are expressed in islet $\alpha$ - and $\beta$-cells, we first determined the specificities of available antibodies to Large Maf proteins by Western blotting. Extracts of HeLa cells transfected with an expression vector for EGFP, MafA, MafB, or c-Maf were separated by SDS-PAGE and blotted with anti-c-Maf (M-153) (Fig. 2, left panels, top). As expected, all of the MafA, MafB and c-Maf proteins were recognized by this antiserum. Anti-c-Maf (M-153) also reacted with $\mathrm{Nrl}$ (data not shown). The anti-MafA peptide serum described above reacted with MafA, but not with MafB or c-Maf (Fig. 2, second panel). We also raised an antiserum against the MafB-specific peptide, and found that it (anti-MafB) specifically reacted with MafB (Fig. 2, third panel). Moreover, the anti-c-Maf (N-15) antibody (Santa Cruz Biotechnology) selectively reacted with c-Maf (Fig. 2, bottom panel), although it reacts only weakly with c-Maf of mouse origin, probably because it was raised against human c-Maf peptide and the mouse c-Maf epitope contains a single amino acid substitution that differs in human c-Maf (data not shown).

We subsequently used these antibodies to detect Maf proteins expressed in glucagonoma and insulinoma cell lines. Western blot analysis of total cell extracts derived from $\alpha \mathrm{TCl}$ (glucagonoma) and 3 TC6 (insulinoma) cells with anti-c-Maf (M-153) indicated that both cell types express Large Maf proteins (Fig. 2, right panels, top). As was the case for HeLa cell transfectants, it was quite difficult to distinguish MafA, MafB and c-Maf by their apparent molecular masses (approximately $50 \mathrm{kDa}$ ) in SDS-PAGE, because they are phosphorylated on multiple sites (Benkhelifa et al. 2001). It was possible to distinguish $\mathrm{Nrl}$, since it migrates faster (approximately $30 \mathrm{kDa}$ ) when transfected into HeLa cells (data not shown). However, in aTC1 and $\beta$ TC6 cells, we did not detect crossreacting protein species of this molecular mass (data not shown). Next, by using specific antisera, we found that MafA is expressed in $\beta$ TC6 cells but not aTG1 (Fig. 2, second panel) and that MafB is expressed in neither cell line (Fig. 2, third panel). Using anti-c-Maf (N-15), we found that $\alpha \mathrm{TC} 1$ cells specifically express c-Maf (Fig. 2, bottom panel), but we also detected a marginal amount of c-Maf in $\beta$ TC6 cells (Fig. 2, bottom panel).

We further used these antibodies to immunostain HeLa cells which had been transiently transfected with an expression vector for MafA, MafB or c-Maf (Fig. 3A). This indicated that anti-c-Maf (M-153) reacted with MafA, MafB and c-Maf, whereas anti-MafA, anti-MafB and anti-cMaf (N-15) sera were specific to MafA, MafB and c-Maf respectively.

Furthermore, we stained $\alpha \mathrm{TC} 1$ and $\beta$ TC6 cells with these sera (Fig. 3B). Nuclear staining of $\alpha \mathrm{TC} 1$ 

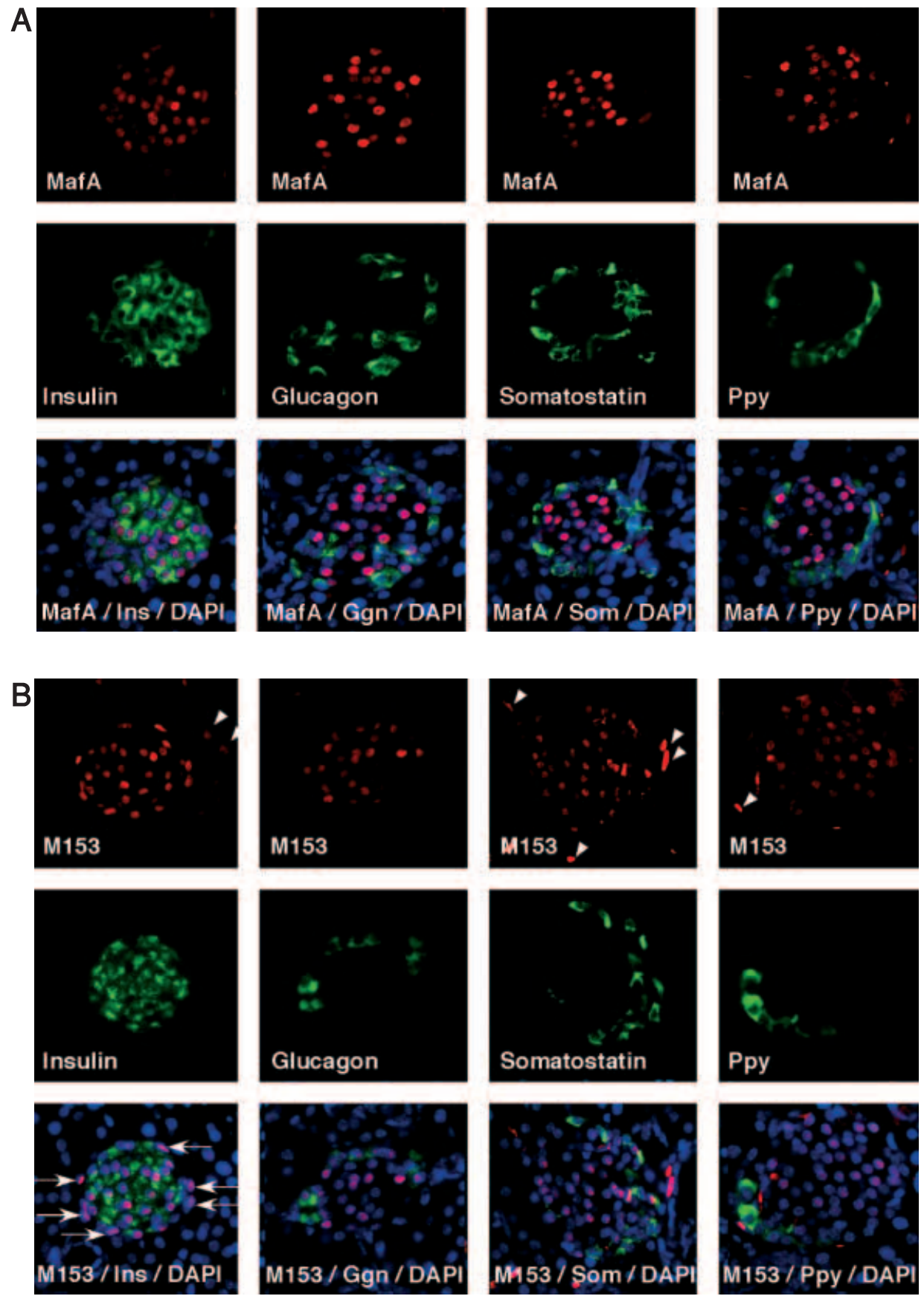

Figure 1 Differential expression of Large Maf proteins in islets of the pancreas. Sections of adult mouse pancreas were double-stained with (A) anti-MafA or (B) anti-pan-Large Maf (anti-c-Maf (M-153)) (red, top panels) serum and with anti-insulin (Ins), anti-glucagon (Ggn), somatostatin (Som) or anti-pancreatic polypeptide (Ppy) serum (green, second panels). DNA was also stained with

4,6-diamidino-2-phenylindole (DAPI) (blue, bottom panels). All the MafA-expressing cells were positive for staining of insulin $(A)$, whereas anti-c-Maf $(M-153)$ serum stained both insulin- and glucagon-expressing cells (B). Arrowheads in panel B indicate Maf-positive non-islet cells, and arrows indicate Maf-positive and insulin-negative cells. 


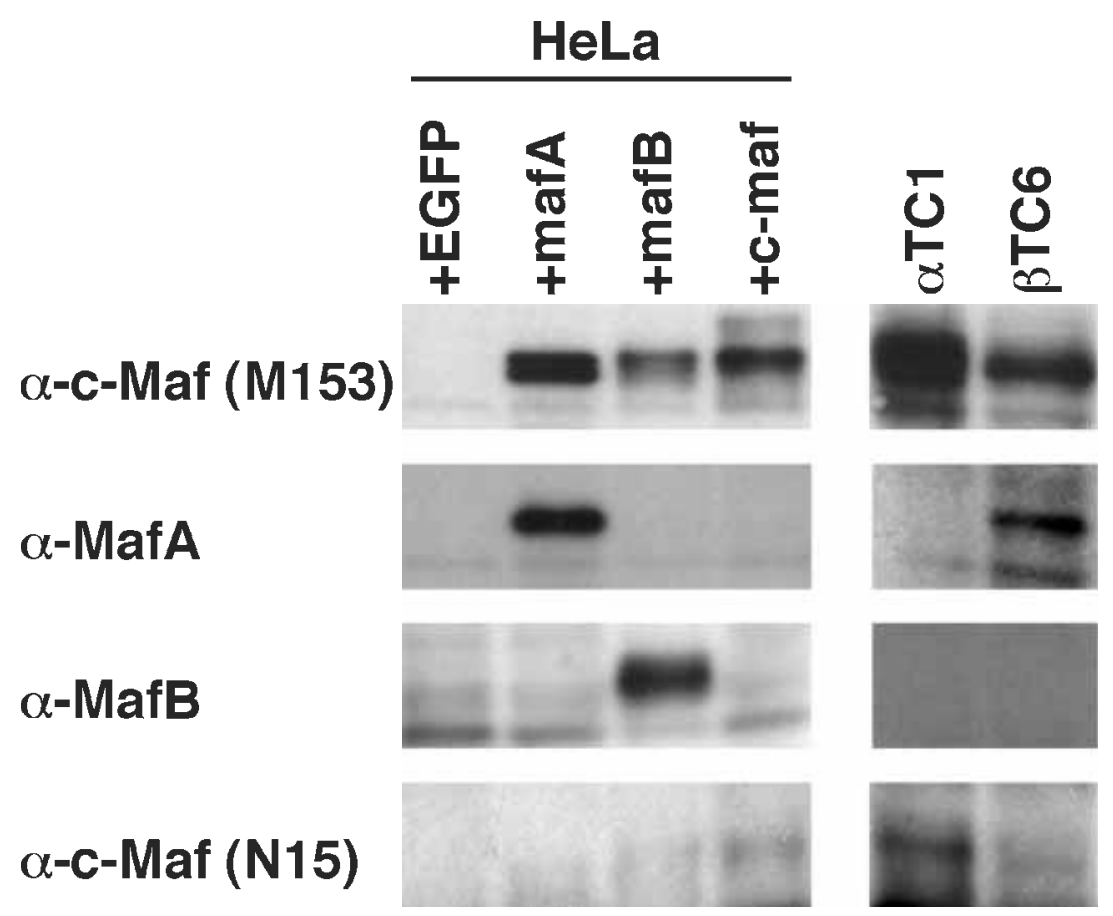

Figure 2 Expression profiling of Large Maf proteins in glucagonoma and insulinoma cell lines. Whole-cell extracts of $\alpha \mathrm{TC} 1, \beta \mathrm{TC} 6$ (right panels) or HeLa cells transiently expressing EGFP, MafA, MafB or c-Maf (left panels) were separated by $8 \%$ SDS-PAGE followed by Western blotting using anti-c-Maf (M-153), anti-MafA, anti-MafB or anti-c-Maf (N-15) antibodies.

cells was observed using anti-c-Maf (M-153) and anti-c-Maf (N-15), indicating that $\alpha \mathrm{TCl}$ cells express c-Maf. In contrast, anti-c-Maf (M-153) and anti-MafA sera stained the nuclei of $\beta$ TC6 cells. In contrast to the Western blotting data (Fig. 2), anti-c-Maf (N-15) serum produced no signal. This is probably because this antiserum reacts weakly with c-Maf of mouse origin as described above, and/or because the level of c-Maf protein in $\beta$ TC6 cells is below the threshold required for detection by immunofluorescent staining with this antiserum.

We also stained sections of mouse pancreas with anti-MafB and anti-c-Maf (N-15) antisera. Using anti-MafB, we did not observe any signal in pancreas, whereas it did react with lens cell nuclei, where MafB is known to be expressed (Sakai et al. 1997) (data not shown). However, unfortunately, we could not detect positive signals when using anti-c-Maf (N-15) serum, even in lens fiber cells (where c-Maf protein expression would be expected (Kawauchi et al. 1999, Kim et al. 1999, Ring et al. 2000)). This may reflect its low reactivity with mouse c-Maf, as described above, and/or incom- patibility of this antiserum with the section staining procedure.

Taken together, these results indicated that in pancreatic islets (i) MafA is specifically expressed in $\beta$-cells, (ii) c-Maf is expressed in $\alpha$-cells, (iii) $\delta$ - and $\gamma$-cells do not express Large Maf proteins, and (iv) c-Maf is possibly expressed at low levels in $\beta$-cells.

\section{Maf proteins interact with the glucagon and insulin promoters in vivo}

We and others have previously shown that MafA is a transactivator that binds to the RIPE3b/MARE element of the insulin promoter (Kataoka et al. 2002, Olbrot et al. 2002, Matsuoka et al. 2003). In addition, Planque et al. (2001) have shown that the Large Maf family members can activate the glucagon promoter by binding to a MARE-like element in the G1 domain of that promoter. Although Matsuoka et al. (2003) have shown that MafA binds to the insulin promoter region in a $\beta$-cell line by a chromatin immunoprecipitation assay, direct evidence that Maf proteins bind to the 
A

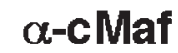
(M153)
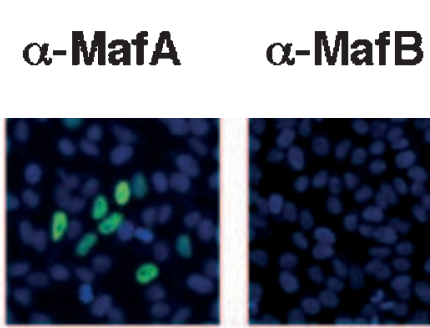

$\alpha$-cMaf (N15)

MafA
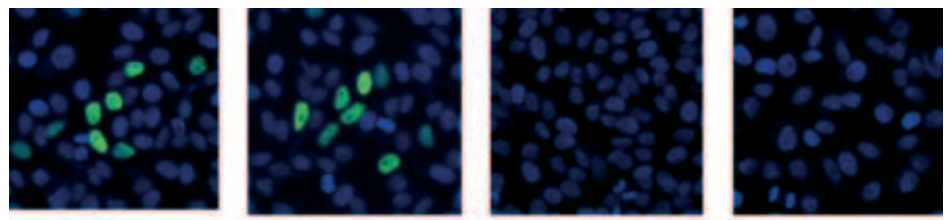

MafB
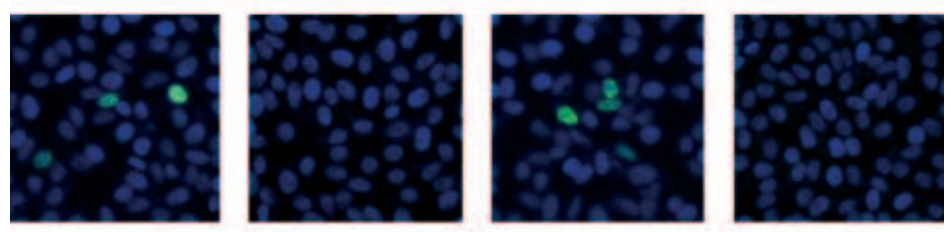

cMaf
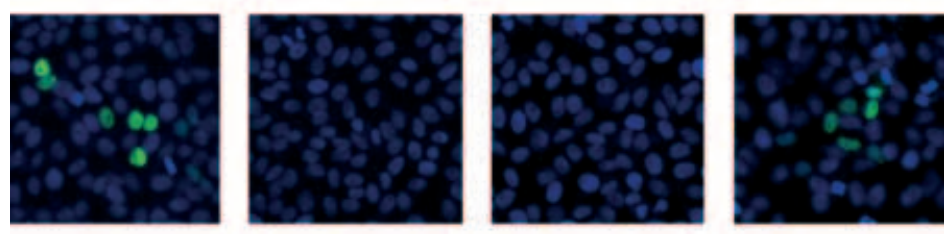

B
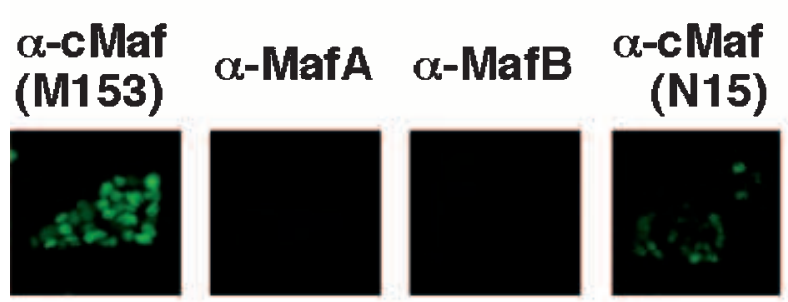

$\alpha \mathrm{TC1}$
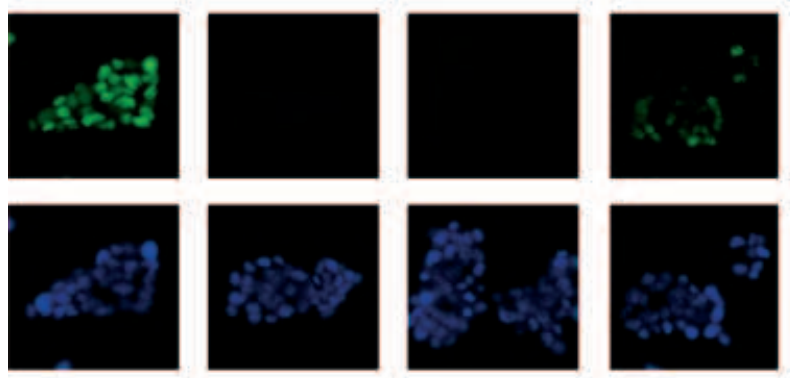

вTC6
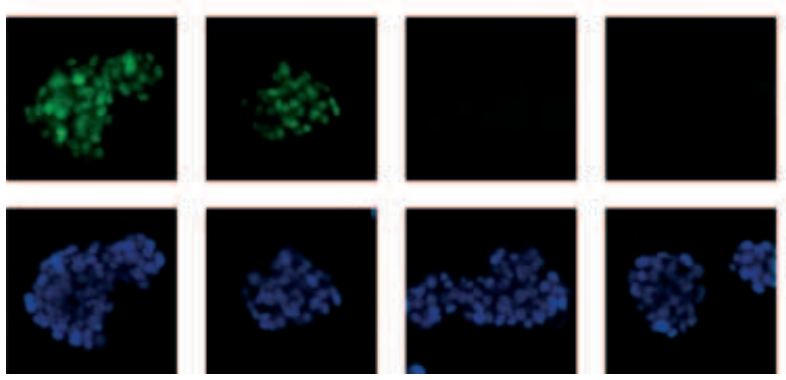

Figure 3 Immunofluorescent staining. HeLa cells transfected with expression vectors for MafA, MafB or c-Maf (A), or $\alpha \mathrm{TC} 1$ and $\beta \mathrm{TC} 6$ cells (B) were stained with anti-c-Maf (M-153), anti-MafA, anti-MafB or anti-c-Maf $(\mathrm{N}-15)$ antibodies and Alexa-Fluor 488-labeled secondary antibody (green) and with 4,6-diamidino-2-phenylindole (blue). 


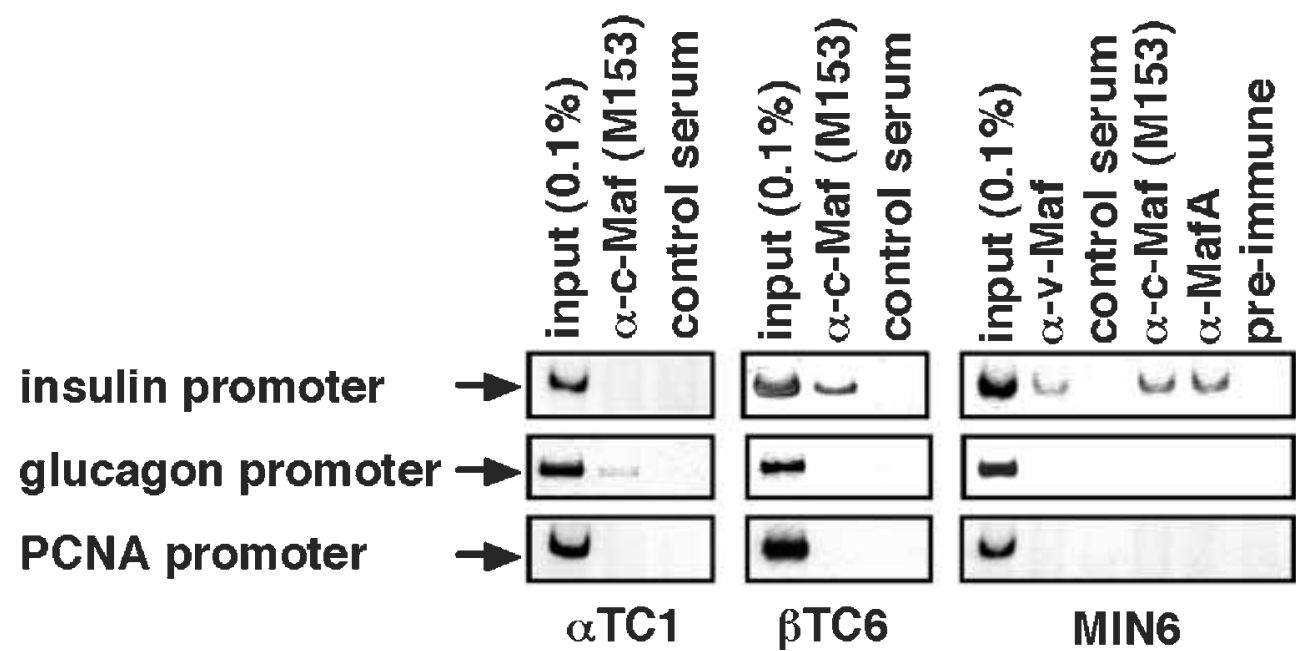

Figure 4 Chromatin immunoprecipitation assay. Cross-linked chromatin isolated from $\alpha$ TC1, $\beta$ TC6, and MIN6 cells was immunoprecipitated with anti-c-Maf (M-153), anti-MafA and anti-v-Maf sera. Anti-GST antibody or anti-MafA preimmune serum were used as negative controls. The precipitated DNA was amplified by PCR using specific primers for promoter regions of insulin, glucagon or PCNA, and was analyzed by PAGE followed by staining with ethidium bromide.

glucagon promoter in vivo is lacking. We therefore examined whether Maf binds to the promoter in $\alpha$ - and $\beta$-cell lines by a chromatin immunoprecipitation assay.

We found that anti-c-Maf (M-153) specifically precipitated the glucagon promoter region from $\alpha \mathrm{TCl}$ cells, but did not precipitate the insulin or unrelated PCNA promoters (Fig. 4). This indicated that c-Maf in $\alpha \mathrm{TCl}$ cells associates specifically with the glucagon promoter. Conversely, this antiserum precipitated the insulin promoter region in 3 TC6 and MIN6 cells, demonstrating that MafA (and possibly c-Maf) specifically interact with the insulin promoter in $\beta$-cell lines. Specific association of MafA with the insulin promoter in MIN6 cells was also evident by using anti-MafA and another pan-Large Maf serum (anti-v-Maf). These results clearly showed that c-Maf in $\alpha$-cells and MafA (and c-Maf) in $\beta$-cells specifically bind to the glucagon and insulin promoters respectively, in vivo.

\section{Activation of the glucagon promoter by c-Maf}

We have previously showed that MafA alone is a much stronger transactivator for the insulin promoter than the other insulin promoter transcription factors, Pdxl and Beta2 (Kataoka et al.
2002). We therefore compared the transcriptional activity of c-Maf with other transcription factors that have been shown to be involved in transcription of the glucagon gene, such as Cdx2, Pax6 and Isl- $1 \alpha$, and their splice variants Pax6-5a and Isl-1 $\beta$. Transfection of the glucagon promoterluciferase reporter into fibroblast cells (NIH3T3) with an expression vector for c-Maf resulted in a great increase in luciferase activity (Fig. 5). When the MARE-like sequence of the promoter was mutated, this activation was greatly reduced (data not shown). We also found that c-Maf was the most efficient transactivator for the glucagon promoter among these $\alpha$-cell-enriched transcription factors (Fig. 5).

In order to evaluate the role of Maf proteins in glucagon gene transcription in $\alpha$-cells, we designed shRNA expression vectors targeted to inhibit c-Maf or MafB expression. Specificity of these shRNAs was verified by co-transfection into NIH3T3 cells (Fig. 6A). Expression of c-Maf and MafB proteins was specifically interfered with by $c-m a f$ and $m a f B$ shRNAs respectively. We then transfected these shRNA vectors into $\alpha \mathrm{TG} 1$ cells with the glucagon promoter-luciferase reporter. As shown in Fig. 6B (left panel), co-transfection of $c$-maf shRNA resulted in significant decrease of the luciferase activity, whereas mafB shRNA had no effect. Furthermore, 


\section{m-glucagon-promoter-luc}

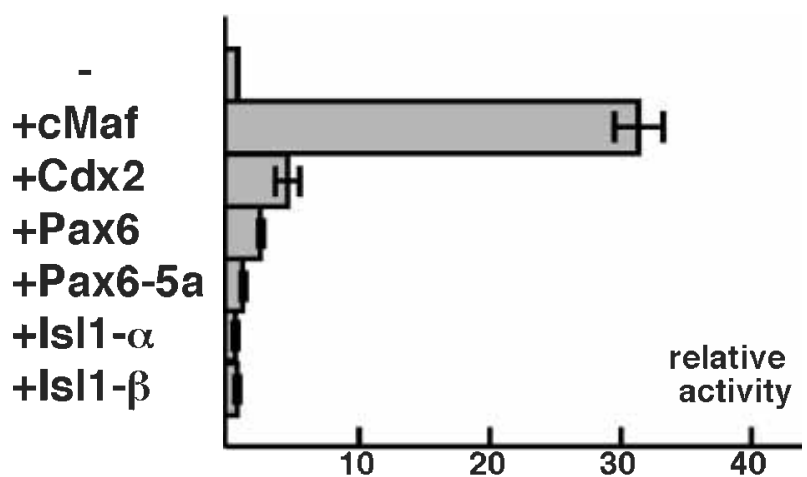

Figure 5 Activation of the glucagon promoter by $\alpha$-cell-enriched transcription factors. NIH3T3 cells were transfected with $100 \mathrm{ng}$ luciferase plasmid driven from glucagon promoter and $100 \mathrm{ng}$ expression plasmids for c-Maf, Cdx2, Pax6, Pax6-5a, Isl1- $\alpha$ or Isl1- $\beta$.

Twenty-four hours after transfection, cells were harvested and luciferase activity was measured. Data are expressed as mean values \pm S.E. of two independent experiments.

activity of control EF1 $\alpha$ (elongation factor $1 \alpha$ ) promoter was not affected by these shRNAs (Fig. 6B right panel). We also found that mutation in the MARE-like sequence in the glucagon promoter resulted in a marked reduction in its activity in $\alpha$ TC1 cells (data not shown). These results strongly support the idea that binding of c-Maf in $\alpha$-cells to the MARE sequence within the glucagon promoter is necessary for its full promoter activity.

\section{Discussion}

In this report, by defining specificities of available anti-Maf antibodies, we provide a demonstration of endocrine cell-type-specific distribution of Large Maf family members in pancreatic islets. MafA is specifically expressed in $\beta$-cells, and c-Maf in $\alpha$-cells. These expression profiles are quite similar for both glucagonoma and insulinoma cell lines. We also found that $\beta$-cells possibly express a small amount of c-Maf. We further provide direct evidence that c-Maf and MafA bind to the promoter of the cell-type-specific hormone genes, glucagon and insulin, respectively, and activate their expression.

Recently, Matsuoka et al. (2003) have also reported that MafA is expressed in $\beta$-cells of islets.
They also demonstrated that MafB is expressed in $\alpha$-cells and in a small fraction of $\beta$-cells of mouse islets by immunostaining. However, in our investigation, MafB protein was not detected in islets of the pancreas. This discrepancy may come from the different anti-MafB sera used in these two studies, and expression of $\mathrm{MafB}$ and its role in pancreatic islets needs to be examined carefully. In this study, we showed that c-Maf, but not MafB, is expressed in $\alpha \mathrm{TC} 1$ cells, although both c-maf and mafB mRNA were expressed (K Kataoka, unpublished observations). We also showed that c-maf shRNA, but not mafB shRNA, affected the glucagon promoter activity in $\alpha \mathrm{TCl}$ cells. Therefore, regulation of $\mathrm{MafB}$ expression at a posttranscriptional level, such as control of translation and/or protein stability, may exist, and thus MafB protein may be expressed in endocrine cells during the development of pancreatic islets.

It has been shown that L-Maf (the chicken homologue of MafA), MafB and c-Maf are expressed in lens cells in similar, but different, spatio-temporal patterns, where they regulate expression of lens-specific crystallin genes and lens development (Ogino \& Yasuda 2000). For example, in chicken, L-Maf expression is seen initially in lens placode and persists during lens fiber cell differentiation (Ogino \& Yasuda 1998). In rodents, c-Maf is expressed in lens fiber cells, whereas MafB is expressed in lens epithelial cells (Sakai et al. 1997, Kawauchi et al. 1999, Kim et al. 1999, Ring et al. 2000). On the contrary, $\mathrm{Nrl}$ is specifically expressed in rod cells of neural retina, and regulates rhodopsin transcription and retinal development (Mears et al. 2001). This kind of expression pattern and gene regulation by Large Maf members is analogous to our finding that MafA and c-Maf are expressed in different endocrine cells $(\beta$ - and $\alpha$-cells) of islets and regulate insulin and glucagon expression. As far as we have determined, endocrine $\delta$ - and $\gamma$-cells do not express Large Mafs, but there remains the possibility that these cells express Maf members in some stages of development.

Insofar as we have determined, c-Maf is the strongest transactivator of the glucagon promoter among the other $\alpha$-cell-expressed transcription factors, Cdx2, Pax6 and Isl-1. We previously found that MafA was a much stronger transactivator of the insulin promoter than two other $\beta$-cell-specific transcription factors, $\mathrm{Pdx} 1$ and Beta2, and that no synergistic transactivation was observed between 


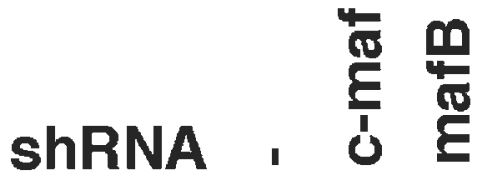
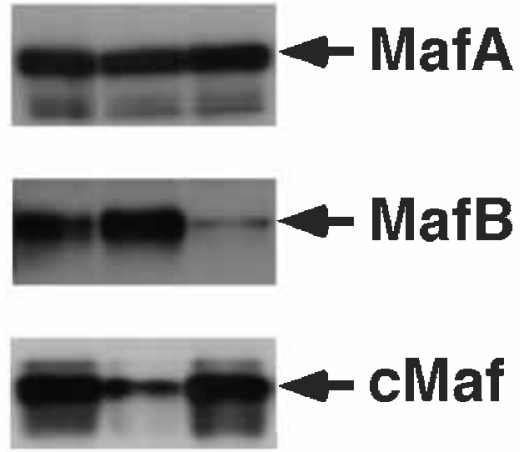

\section{m-glucagon -promoter-Iuc \\ EF1 $\alpha$-luc}

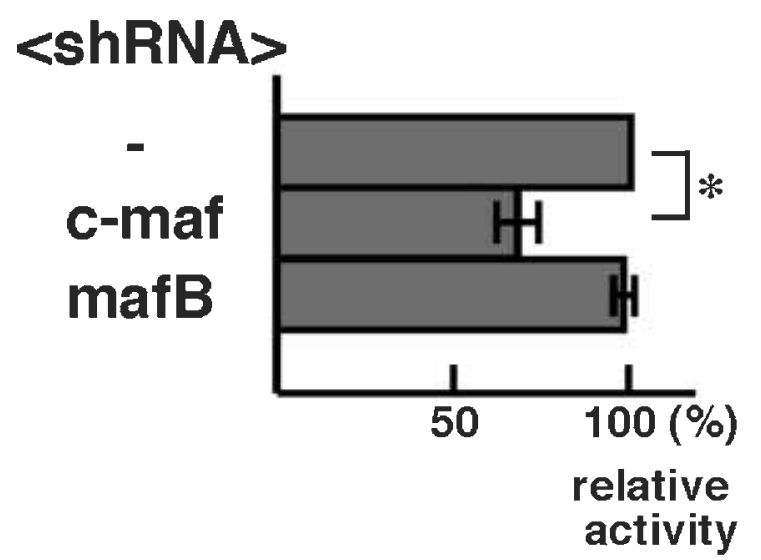

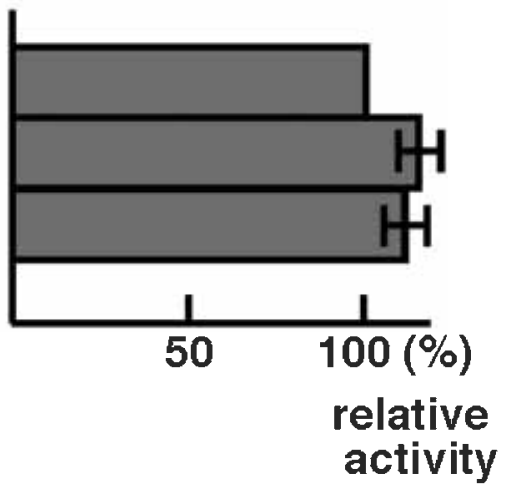

Figure 6 RNA interference. (A) Specificity of shRNAs. NIH3T3 cells were transfected with $0.1 \mu \mathrm{g}$ expression plasmids for MafA, MafB or c-Maf and $1.5 \mu \mathrm{g}$ shRNA vector driven by U6 small nuclear RNA promoter. Whole-cell extracts were prepared $24 \mathrm{~h}$ after transfection and were analyzed by Western blotting using anti-c-Maf (M-153) serum. (B) Luciferase reporter plasmid $(0.1 \mu \mathrm{g})$ driven from the mouse glucagon promoter (left) or EF1 $\alpha$ promoter (right) was transfected into $\alpha \mathrm{TC} 1$ cells with shRNA vector constructs $(1.4 \mu \mathrm{g})$. Relative luciferase activities are shown as mean values \pm S.E. of five (c-maf) or three (mafB) independent experiments. Statistical significance was calculated by Student's $t$-test. ${ }^{*} P<0.005$.

MafA and Pdx1 or Beta2 (Kataoka et al. 2002). Synergistic activation of the glucagon promoter by Large Maf proteins and Pax6 has been reported previously (Planque et al. 2001), but we did not find any synergy between c-Maf and Cdx2, Pax6 or Isl-1 in our transient transfection assays which employed NIH3T3 cells (K Kataoka, unpublished observations). 
Recently, MafA was shown to bind and activate a $\beta$-cell-specific enhancer element of the $\mathrm{Pdxl}$ transcription factor (Samaras et al. 2003). Therefore, MafA is possibly involved in regulating other $\beta$-cell-specific genes such as GLUT2 and glucokinase. Similarly, c-Maf may also regulate other $\alpha$-cellspecific genes. Many transcription factors have been identified to date that are involved in insulin or glucagon transcription, and many of them are shown to be also necessary for $\beta$ - and $\alpha$-cell development respectively (St-Onge et al. 1999, Dohrmann et al. 2000, Schwitzgebel 2001). Thus, MafA and c-Maf may also play important roles in the development of these endocrine cells. Generation of a mafA knockout mouse and investigation of its $\beta$-cell development and insulin secretion may help to elucidate this possibility. To date, there have been no reports of defective $\alpha$-cell development or glucagon secretion in c-maf knockout mice (Kawauchi et al. 1999, Kim et al. 1999, Ring et al. 2000), but results we present may justify a re-examination of these models. It will also be important to determine the expression profiles of c-Maf, MafA and MafB during normal pancreatic development and in mice that show defects in islet cell development such as $p d x 1$, beta2 and pax6 knockouts. These approaches may ultimately help to elucidate the genetic cascade involved in the regulation of islet cell development.

\section{Acknowledgements}

We thank Dr Yuji Kageyama and Dr Hasan Mahmud Reza for helpful discussions. We are also grateful to Jun-ichi Miyazaki (Osaka University) for the MIN6 cell line, Dr Makoto Nishizawa for recombinant genomic clones of mouse mafB (pMmb32) and human c-maf (pHM-3), Dr Noriyuki Azuma (National Children's Hospital, Tokyo) and Dr Yuki Yamaguchi (Tokyo Institute of Technology, Yokohama) for pCAGGS/Pax6 and pCAGGS/Pax6-5a plasmids. This work was supported by Grants-in-Aid for Scientific Research on Priority Areas, for Encouragement of Young Scientists, and of the 21st Century COE Program from the Ministry of Education, Culture, Sports, Science and Technology in Japan, and a grant from the Ministry of Health, Labor and Welfare in Japan to K K.

\section{References}

Ando K, Shioda S, Handa H \& Kataoka K 2003 Isolation and characterization of an alternatively spliced variant of transcription factor Islet-1. Fournal of Molecular Endocrinology 31 419-425.

Benkhelifa S, Provot S, Nabais E, Eychene A, Calothy G \& Felder-Schmittbuhl MP 2001 Phosphorylation of MafA is essential for its transcriptional and biological properties. Molecular and Cellular Biology 21 4441-4452.

Boyd KE, Wells J, Gutman J, Bartley SM \& Farnham PJ 1998 c-Myc target gene specificity is determined by a post-DNA binding mechanism. PNAS 95 13887-13892.

Crowe DT \& Tsai MJ 1989 Mutagenesis of the rat insulin II 5 '-flanking region defines sequences important for expression in HIT cells. Molecular and Cellular Biology 9 1784-1789.

Dohrmann C, Gruss P \& Lemaire L 2000 Pax genes and the differentiation of hormone-producing endocrine cells in the pancreas. Mechanisms of Development 92 47-54.

Drucker DJ, Philippe J, Jepeal L \& Habener JF 1987 Glucagon gene $5^{\prime}$-flanking sequences promote islet cell-specific gene transcription. Journal of Biological Chemistry 262 15659-15665.

Jin T \& Drucker DJ 1996 Activation of proglucagon gene transcription through a novel promoter element by the caudal-related homeodomain protein cdx-2/3. Molecular and Cellular Biology 16 19-28.

Karlsson O, Edlund T, Moss JB, Rutter WJ \& Walker MD 1987 A mutational analysis of the insulin gene transcription control region: expression in beta cells is dependent on two related sequences within the enhancer. PNAS 84 8819-8823.

Kataoka K, Nishizawa M \& Kawai S 1993 Structure-function analysis of the maf oncogene product, a member of the b-Zip protein family. Fournal of Virology 67 2133-2141.

Kataoka K, Fujiwara KT, Noda M \& Nishizawa M 1994a MafB, a new Maf family transcription activator that can associate with Maf and Fos but not with Jun. Molecular and Cellular Biology 14 7581-7591.

Kataoka K, Noda M \& Nishizawa M $1994 b$ Maf nuclear oncoprotein recognizes sequences related to an AP-1 site and forms heterodimers with both Fos and Jun. Molecular and Cellular Biology 14 700-712.

Kataoka K, Han SI, Shioda S, Hirai M, Nishizawa M \& Handa H 2002 MafA is a glucose-regulated and pancreatic $\beta$-cell-specific transcriptional activator for the insulin gene. Fournal of Biological Chemistry 277 49903-49910.

Kawai S, Goto N, Kataoka K, Saegusa T, Shinno-Kohno H \& Nishizawa M 1992 Isolation of the avian transforming retrovirus, AS42, carrying the $\mathrm{v}$-maf oncogene and initial characterization of its gene product. Virology 188 778-784.

Kawauchi S, Takahashi S, Nakajima O, Ogino H, Morita M, Nishizawa M, Yasuda K \& Yamamoto M 1999 Regulation of lens fiber cell differentiation by transcription factor c-Maf. Fournal of Biological Chemistry 274 19254-19260.

Kim JI, Li T, Ho IC, Grusby MJ \& Glimcher LH 1999 Requirement for the c-Maf transcription factor in crystallin gene regulation and lens development. PNAS 96 3781-3785.

Knepel W, Jepeal L \& Habener JF 1990 A pancreatic islet cell-specific enhancer-like element in the glucagon gene contains two domains binding distinct cellular proteins. Fournal of Biological Chemistry 265 8725-8735.

Knepel W, Vallejo M, Chafitz JA \& Habener JF 1991 The pancreatic islet-specific glucagon G3 transcription factors recognize control elements in the rat somatostatin and insulin-I genes. Molecular Endocrinology 5 1457-1466.

Laser B, Meda P, Constant I \& Philippe J 1996 The caudal-related homeodomain protein $\mathrm{Cdx}-2 / 3$ regulates glucagon gene expression in islet cells. Fournal of Biological Chemistry $27 \mathbf{1}$ 28984-28994. 
Marshak S, Totary H, Cerasi E \& Melloul D 1996 Purification of the $\beta$-cell glucose-sensitive factor that transactivates the insulin gene differentially in normal and transformed islet cells. PNAS 93 15057-15062.

Matsuoka T, Zhao L, Artner I, Jarrett HW, Friedman D, Means A \& Stein R 2003 Members of the large Maf transcription family regulate insulin gene transcription in islet $\beta$ cells. Molecular and Cellular Biology 23 6049-6062.

Mears AJ, Kondo M, Swain PK, Takada Y, Bush RA, Saunders TL, Sieving PA \& Swaroop A $2001 \mathrm{Nrl}$ is required for rod photoreceptor development. Nature Genetics 29 447-452.

Melloul D, Marshak S \& Cerasi E 2002 Regulation of insulin gene transcription. Diabetologia 45 309-326.

Miyazaki J, Araki K, Yamato E, Ikegami H, Asano T, Shibasaki Y, Oka Y \& Yamamura K 1990 Establishment of a pancreatic $\beta$ cell line that retains glucose-inducible insulin secretion: special reference to expression of glucose transporter isoforms. Endocrinology 127 126-132.

Morel C, Cordier-Bussat M \& Philippe J 1995 The upstream promoter element of the glucagon gene, G1, confers pancreatic alpha cell-specific expression. Fournal of Biological Chemistry 270 3046-3055.

Naya FJ, Stellrecht CM \& Tsai MJ 1995 Tissue-specific regulation of the insulin gene by a novel basic helix-loop-helix transcription factor. Genes and Development 9 1009-1019.

Ogino H \& Yasuda K 1998 Induction of lens differentiation by activation of a bZIP transcription factor, L-Maf. Science $\mathbf{2 8 0}$ $115-118$.

Ogino H \& Yasuda K 2000 Sequential activation of transcription factors in lens induction. Development Growth and Differentiation 42 437-448.

Ohlsson H, Karlsson K \& Edlund T 1993 IPF1, a homeodomain-containing transactivator of the insulin gene. EMBO Fournal 12 4251-4259.

Ohneda K, Ee H \& German M 2000 Regulation of insulin gene transcription. Seminars in Cell and Developmental Biology 11 227-233.

Olbrot M, Rud J, Moss LG \& Sharma A 2002 Identification of beta-cell-specific insulin gene transcription factor RIPE3b1 as mammalian MafA. PNAS 99 6737-6742.

Petersen HV, Serup P, Leonard J, Michelsen BK \& Madsen OD 1994 Transcriptional regulation of the human insulin gene is dependent on the homeodomain protein STF1/IPF1 acting through the CT boxes. PNAS 91 10465-10469.

Philippe J, Drucker DJ, Knepel W, Jepeal L, Misulovin Z \& Habener JF 1988 Alpha-cell-specific expression of the glucagon gene is conferred to the glucagon promoter element by the interactions of DNA-binding proteins. Molecular and Cellular Biology 8 4877-4888.
Planque N, Leconte L, Coquelle FM, Benkhelifa S, Martin P, Felder-Schmittbuhl MP \& Saule S 2001 Interaction of Maf transcription factors with Pax-6 results in synergistic activation of the glucagon promoter. Fournal of Biological Chemistry 276 35751-35760.

Ring BZ, Cordes SP, Overbeek PA \& Barsh GS 2000 Regulation of mouse lens fiber cell development and differentiation by the Maf gene. Development 127 307-317.

Ritz-Laser B, Estreicher A, Klages N, Saule S \& Philippe J 1999 Pax-6 and Cdx-2/3 interact to activate glucagon gene expression on the G1 control element. Fournal of Biological Chemistry 274 $4124-4132$.

Sakai M, Imaki J, Yoshida K, Ogata A, Matsushima-Hibaya Y, Kuboki Y, Nishizawa M \& Nishi S 1997 Rat maf related genes: specific expression in chondrocytes, lens and spinal cord. Oncogene $14745-750$

Samaras SE, Zhao L, Means A, Henderson E, Matsuoka TA \& Stein R 2003 The islet $\beta$ cell-enriched RIPE3bl/Maf transcription factor regulates $p d x-1$ expression. Fournal of Biological Chemistry 278 12263-12270.

Schwitzgebel VM 2001 Programming of the pancreas. Molecular and Cellular Endocrinology 185 99-108.

Shieh SY \& Tsai MJ 1991 Cell-specific and ubiquitous factors are responsible for the enhancer activity of the rat insulin II gene. Journal of Biological Chemistry 266 16708-16714.

St-Onge L, Wehr R \& Gruss P 1999 Pancreas development and diabetes. Current Opinion in Genetics and Development 9 295-300.

Sui G, Soohoo G, Affar EB, Gay F, Shi Y, Forrester WC \& Shi Y 2002 A DNA vector-based RNAi technology to suppress gene expression in mammalian cells. PNAS 99 5515-5520.

Swaroop A, Xu JZ, Pawar H, Jackson A, Skolnick C \& Agarwal N 1992 A conserved retina-specific gene encodes a basic motif/leucine zipper domain. PNAS 89 266-270.

Wang M \& Drucker DJ 1995 The LIM domain homeobox gene isl-1 is a positive regulator of islet cell-specific proglucagon gene transcription. Fournal of Biological Chemistry 270 12646-12652.

Whelan J, Poon D, Weil PA \& Stein R 1989 Pancreatic beta-cell-type-specific expression of the rat insulin II gene is controlled by positive and negative cellular transcriptional elements. Molecular and Cellular Biology 9 3253-3259.

Yamaguchi Y, Sawada J, Yamada M, Handa H \& Azuma N 1997 Autoregulation of Pax6 transcriptional activation by two distinct DNA-binding subdomains of the paired domain. Genes to Cells $\mathbf{2}$ 255-261.

Received 22 September 2003

Accepted 29 October 2003 